\title{
Electroacupuncture ameliorates cognitive impairment through inhibition of NF-kB-mediated neuronal cell apoptosis in cerebral ischemia-reperfusion injured rats
}

\author{
XIAODONG FENG ${ }^{1}$, SHANLI YANG ${ }^{2,3}$, JIAO LIU $^{5}$, JIA HUANG ${ }^{1}$, JUN PENG $^{4}$, \\ JIUMAO LIN ${ }^{4}$, JING TAO ${ }^{1-3}$ and LIDIAN CHEN ${ }^{1-3}$ \\ ${ }^{1}$ College of Rehabilitation Medicine; ${ }^{2}$ MOE Key Laboratory of Traditional Chinese Medicine on \\ Osteology and Traumatology and Exercise Rehabilitation; ${ }^{3}$ Fujian Key Laboratory of Exercise Rehabilitation; \\ ${ }^{4}$ Academy of Integrative Medicine, Fujian University of Traditional Chinese Medicine, Fuzhou, Fujian 350122; \\ ${ }^{5}$ Henan College of Traditional Chinese Medicine, Zhengzhou, Henan 450008, P.R. China
}

Received December 4, 2012; Accepted March 15, 2013

DOI: $10.3892 / \mathrm{mmr} .2013 .1392$

\begin{abstract}
Cognitive impairment is a serious mental deficit following stroke that severely affects the quality of life of stroke survivors. Nuclear factor- $\kappa \mathrm{B}(\mathrm{NF}-\kappa \mathrm{B})$-mediated neuronal cell apoptosis is involved in the development of post-stroke cognitive impairment; therefore, it has become a promising target for the treatment of impaired cognition. Acupuncture at the Baihui (DU20) and Shenting (DU24) acupoints is commonly used in China to clinically treat post-stroke cognitive impairment; however, the precise mechanism of its action is largely unknown. In the present study, we evaluated the therapeutic efficacy of electroacupuncture against post-stroke cognitive impairment and investigated the underlying molecular mechanisms using a rat model of focal cerebral ischemia-reperfusion (I/R) injury. Electroacupuncture at Baihui and Shenting was identified to significantly ameliorate neurological deficits and reduce cerebral infarct volume. Additionally, electroacupuncture improved learning and memory ability in cerebral I/R injured rats, demonstrating its therapeutic efficacy against post-stroke cognitive impairment. Furthermore, electroacupuncture significantly suppressed the I/R-induced activation of
\end{abstract}

Correspondence to: Dr Jing Tao or Dr Lidian Chen, College of Rehabilitation Medicine, Fujian University of Traditional Chinese Medicine, 1 Huatuo Road, Minhou Shangjie, Fuzhou, Fujian 350122, P.R. China

E-mail: taojing01@yahoo.com.cn

E-mail: cld@fjtcm.edu.cn

Abbreviations: $\mathrm{NF}-\kappa \mathrm{B}$, nuclear factor $\kappa \mathrm{B} ; \mathrm{I} / \mathrm{R}$, ischemia-reperfusion; MCAO, middle cerebral artery occlusion; TTC, 2,3,5-triphenyl tetrazolium chloride; TUNEL, terminal deoxynucleotidyl-transferasemediated dUTP nick end labeling

Key words: electroacupuncture, Baihui (DU20) and Shenting (DU24) acupoints, cognitive impairment, cerebral ischemia-reperfusion, nuclear factor- $\mathrm{\kappa} B$ pathway, apoptosis
$\mathrm{NF}-\kappa \mathrm{B}$ signaling in ischemic cerebral tissues. The inhibitory effect of electroacupuncture on $\mathrm{NF}-\kappa \mathrm{B}$ activation led to the inhibition of cerebral cell apoptosis. Finally, electroacupuncture markedly downregulated the expression of pro-apoptotic Bax and Fas, two critical downstream target genes of the $\mathrm{NF}-\kappa \mathrm{B}$ pathway. Collectively, our findings suggest that inhibition of NF- $\mathrm{NB}$-mediated neuronal cell apoptosis may be one mechanism via which electroacupuncture at Baihui and Shenting exerts a therapeutic effect on post-stroke cognitive impairment.

\section{Introduction}

Cognitive impairment is a condition characterized by mental deficits. The most common types of cognitive deficits include attention and language syntax disturbances, delayed recall and executive dysfunction, which lead to difficulties with analysis, interpretation, planning, organization, concentration and other reductions in cognitive functions that severely affect quality of life (1-4). Stroke is one of the most common causes of cognitive impairment (5-8). Approximately $25 \%$ of patients present with cognitive impairment 3 months after a stroke. Furthermore, up to $75 \%$ of stroke survivors may be considered to have cognitive impairment when selective types of cognitive impairment, commonly involving memory, orientation, language and attention, are taken into account (9-11).

Although the pathogenic mechanisms of stroke and post-stoke disabilities are complex, apoptosis has been suggested to be one of the key elements in brain injury following ischemic stroke (12-14). Apoptosis is triggered by intrinsic or extrinsic stimuli. Intrinsic and extrinsic signals eventually lead to the activation of caspases and nucleases, resulting in the destruction of a cell $(15,16)$. The process of apoptosis is highly controlled by a diverse range of intracellular pathways, including nuclear factor $-\kappa \mathrm{B}(\mathrm{NF}-\kappa \mathrm{B})$ signaling. NF- $\kappa \mathrm{B}$, one of the most important nuclear transcription factors, is involved in the regulation of numerous critical physiological processes. In unstimulated cells, $\mathrm{NF}-\kappa \mathrm{B}$ is sequestered in the cytosol via interaction with inhibitory $\mathrm{I} \kappa \mathrm{B}$ proteins. Under pathological conditions, I $\mathrm{B}$ B is phosphorylated by $\mathrm{I} \kappa \mathrm{B}$ kinase (IKK), which 
results in the ubiquitination and degradation of $\mathrm{I} \kappa \mathrm{B}$ proteins and leads to the release of sequestered NF- $\kappa$ B. Following activation, $\mathrm{NF}-\kappa \mathrm{B}$ translocates to the nucleus, where it regulates the expression of various critical genes involved in apoptosis. $\mathrm{NF}-\kappa \mathrm{B}$ has been suggested to play a bi-functional role in the death and survival of neuronal cells (17). Although a number of studies show that NF- $\mathrm{BB}$ activation prevents neuronal cells from undergoing apoptosis $(18,19)$, numerous other studies have suggested that $\mathrm{NF}-\kappa \mathrm{B}$ may have a causative role in excitotoxicity (20-23). In addition, $\mathrm{NF}-\kappa \mathrm{B}$ has been reported to be activated in a cognitive impairment model following stroke, where $\mathrm{NF}-\kappa \mathrm{B}$ inhibitors were shown to significantly improve cognitive function (24). Therefore, suppression of the NF- $\kappa \mathrm{B}$ pathway may be a promising approach for the treatment of ischemic stroke and post-stroke disabilities.

Acupuncture, a medicinal methodology originating from ancient China, has been used for thousands of years in several oriental countries to treat various diseases (25). The clinical efficacy of acupuncture in stroke and post-stroke cognitive impairment has been previously demonstrated (26-29). In the system of traditional Chinese medicine (TCM), Baihui (DU20) and Shenting (DU24) are acupoints that belong to the Du Meridian and may be important in the nervous system. Acupoint Shenting (DU24) is considered to be involved in the improvement of human health and spirits, and Baihui (DU20) in the adjustment of memory function. Therefore, the Baihui and Shenting acupoints are commonly used in China to clinically treat post-stroke cognitive impairment $(30,31)$. However, the precise mechanism of its effect on impaired cognition remains to be elucidated. In the present study, we evaluated the therapeutic efficacy of electroacupuncture against post-stroke cognitive impairment and investigated the underlying molecular mechanisms using a focal cerebral ischemia-reperfusion (I/R)-injured rat model.

\section{Materials and methods}

Materials and reagents. Reverse transcriptase and a TUNEL assay kit were provided by Promega (Madison, WI, USA). TRIzol reagent was purchased from Invitrogen (Carlsbad,

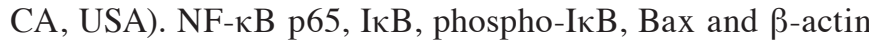
antibodies and horseradish peroxidase (HRP)-conjugated secondary antibodies were obtained from Cell Signaling Technology, Inc. (Beverly, MA, USA). Fas antibody was obtained from Abcam (Cambridge, UK). 2,3,5-Triphenyl tetrazolium chloride (TTC) and all the additional chemicals used were purchased from Sigma Chemicals (St. Louis, MO, USA), unless otherwise stated.

Animals. Male Sprague-Dawley rats (weight, 250-280 g) were obtained from Shanghai SLAC Laboratory Animal Co., Ltd. (Shanghai, China) and housed under pathogen-free conditions with a 12-h light/dark cycle. The rats had free access to food and water during the experiment. The experiments performed in this study were approved by the Institutional Animal Care and Use Committee of Fujian University of Traditional Chinese Medicine (Fuzhou, China).

Establishment of the cerebral I/R injured rat model. The cerebral I/R injured rat model was established by middle cerebral artery occlusion (MCAO), as previously described by Chen et al (32). Briefly, after the rats were anesthetized with $10 \%$ chloral hydrate (intraperitoneal injection), the left common carotid artery (CCA), the left external carotid artery (ECA) and the internal carotid artery (ICA) were carefully exposed by a midline neck incision. The left middle cerebral artery (MCA) was occluded by introducing an embolus through the ICA. Focal cerebral ischemia started when the tip of the catheter reached the origin of the MCA (18-22 mm). Reperfusion was achieved by removing the thread after $2 \mathrm{~h}$ of occlusion to restore blood supply to the MCA area, then the left CCA and ECA were ligated. The rectal temperature of rats was maintained at $37^{\circ} \mathrm{C}$ throughout the surgical procedure. Following surgery, the rats were allowed to recover in pre-warmed cages.

Animal grouping and electroacupuncture treatment. The rats were randomly divided into 3 groups ( $\mathrm{n}=15 /$ group) as follows: i) Rats of the sham operation control (SC) group underwent neck dissection and coagulation of the ECA without occlusion of the MCA; ii) in rats of the ischemia control (IC) group, blood flow to the left MCA was blocked for $2 \mathrm{~h}$, followed by reperfusion; and iii) the electroacupuncture (EA) group underwent the same treatment of I/R as that used in the IC group. Following recovery from surgery ( $2 \mathrm{~h}$ after $\mathrm{I} / \mathrm{R}$ treatment), rats were administered electroacupuncture for $30 \mathrm{~min}$ daily for 10 days in the EA group. The acupuncture needles (diameter, $0.3 \mathrm{~mm}$ ) were inserted at a depth of 2-3 mm into the Baihui (DU20) and Shenting (DU24) acupoints on the head. Stimulation was then generated using the EA apparatus (Model G6805; SMIF, Shanghai, China) and the stimulation parameters were set as disperse waves of 1 and $20 \mathrm{~Hz}$.

Evaluation of neurological deficit scores. The neurological deficit score was examined in a blinded manner as previously described by Chen et al (32) and scores were determined as follows: Score 0, no neurological deficit; score 1 (failure to fully extend the right forepaw), mild deficits; scores 2 (circling to the right) and 3 (falling to the right), moderate deficits; and score 4 (loss of walking), severe deficits. Rats scoring 0 or 4 were excluded from this experiment.

Measurement of cerebral infarct volume. Following completion of the experiment, rats were anesthetized with $10 \%$ chloral hydrate (intraperitoneal injection). Each rat was transcardially perfused with $0.9 \% \mathrm{NaCl}$ and the brain was removed. The brain of each rat was sectioned into $2-\mathrm{mm}$ coronal slices. The slices were stained with $2 \%$ TTC solution (Sigma, St. Louis, $\mathrm{MO}, \mathrm{USA}$ ) at $37^{\circ} \mathrm{C}$ for $20 \mathrm{~min}$ and then fixed with $10 \%$ buffered formalin solution. Images of the stained slices were captured using a high-resolution digital camera (Canon SX20; Canon, Tokyo, Japan) and the infarct volume was quantified with the Motic Med 6.0 system as a percentage of the total brain volume.

Morris water maze. All the rats were subjected to the Morris water maze task from the 4th day after surgery in order to investigate spatial learning and memory ability. The water maze apparatus (Chinese Academy of Sciences, Beijing, China) consisted of a circular pool (diameter, $120 \mathrm{~cm}$; 
depth, $50 \mathrm{~cm}$ ) filled with water (depth, $30 \mathrm{~cm}$; temperature, $26 \pm 2^{\circ} \mathrm{C}$ ). The tank was theoretically divided into four equal quadrants and a video camera attached to a computer was placed above the center of the tank to record and analyze the rats. A submerged safe platform was located in the pool $(2 \mathrm{~cm}$ below water surface; $6 \mathrm{~cm}$ diameter in a fixed position). Morris water maze tasks mainly include orientation navigation and space exploration trials. During the first set of trials, each rat was placed in the water at each of four equidistant locations to the platform. When the rats arrived at the platform within the $90 \mathrm{sec}$ time restriction and remained on it for $3 \mathrm{sec}$, they were considered to have found the platform and were scored by the time taken/length of the route. When the rats were unable to find the platform within $90 \mathrm{sec}$, they were placed on the platform for $10 \mathrm{sec}$ and the time score was $90 \mathrm{sec}$. The time taken and the length of the route by which each rat found the safe platform was recorded by the computer. The average of the time taken and the length of the route for the four quadrants as the result of each rat was assessed every day. The duration of the first set of trials was five days, with the experiment performed once per day. The second part of the experiment was performed on the 9th day, to examine the time in which rats found the location of the platform within the $90 \mathrm{sec}$ time restriction, which tested their ability to remember the position of the platform. After the trials, the rats were dried thoroughly with a hair drier and returned to their cages.

In situ apoptosis detection using TUNEL staining. The rats were anesthetized and transcardially perfused with $0.9 \% \mathrm{NaCl}$ and $4 \%$ paraformaldehyde through the left ventricle and the brain was removed. The samples were fixed in cold $4 \%$ paraformaldehyde and then cut into into $5-\mu \mathrm{m}$ thick sections. In situ apoptosis was analyzed using the TUNEL assay kit. The nuclei of all the cells were visualized by DAPI staining and the green fluorescence of apoptotic cells was detected using a confocal fluorescence microscope (LSM 710; Carl Zeiss Microscopy, Thornwood, NY, USA). Apoptotic cells were counted at four randomly selected microscopic fields (magnification, x200). The apoptotic rate was calculated as the ratio of green-stained cells to the total number of blue DAPI-stained cells.

Direct immunofluorescence analysis of NF- $\kappa B$ p 65 nuclear translocation. The paraffin sections of brain tissues were treated with microwave heat-induced epitope retrieval. After the specimens were washed three times with phosphate-buffered saline (PBS; $\mathrm{pH}$ 7.4), they were incubated for $1 \mathrm{~h}$ at $37^{\circ} \mathrm{C}$ in a 1:50 dilution of rabbit anti-rat NF- $\mathrm{\kappa B}$ p65 antibody (green). Following incubation, washing was repeated. The nuclei of all the cells were counterstained with DAPI. After three washes with PBS, the tissues were mounted in ProLong Gold Antifade reagent. Images were captured using a confocal fluorescence microscope (LSM 710; magnification, x200).

Western blot analysis. Total proteins were extracted from the infarct cortex and separated by electrophoresis on $12 \%$ SDS-PAGE gels. Proteins were then transferred onto PVDF membranes. The membranes were blocked for $2 \mathrm{~h}$ with $5 \%$ nonfat dry milk at room temperature and detected with rabbit -NF-кB p65, -p-IкB, -Fas, -Bax and - $\beta$-actin antibodies (dilu-

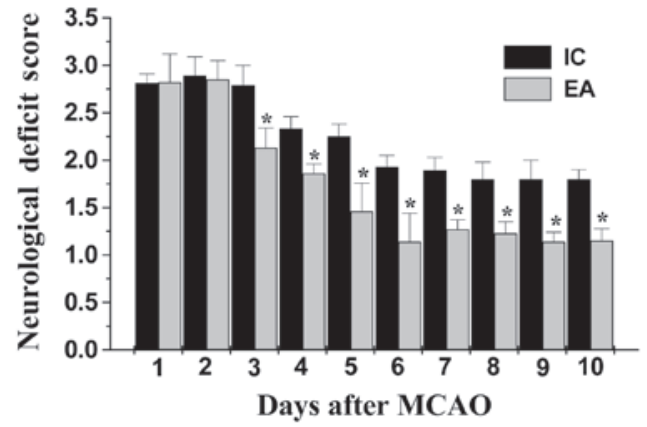

Figure 1. Effect of electroacupuncture at the Baihui (DU20) and Shenting (DU24) acupoints on neurological deficits in cerebral ischemia-reperfusion (I/R)-injured rats. The neurological deficit score was determined. Data are presented as averages with SE (error bars) from 15 individual rats in the electroacupuncture (EA) or ischemia control (IC) groups. ${ }^{*} \mathrm{P}<0.05$ vs. the IC group. MCAO, middle cerebral artery occlusion.

tion, 1:1,000) at $4^{\circ} \mathrm{C}$ overnight, followed by incubation with the appropriate HRP-conjugated secondary antibody for $50 \mathrm{~min}$. The bands were visualized by enhanced chemiluminescence.

RNA extraction and reverse transcription polymerase chain reaction $(R T-P C R)$ analysis. After total RNA was isolated with TRIzol reagent, oligo(dT)-primed RNA $(1 \mu \mathrm{g})$ was reverse transcribed into cDNA according to the manufacturer's instructions. cDNA was used to determine the levels of Fas and Bax mRNA by PCR with Taq DNA polymerase (Fermentas Amherst, NY, USA); $\beta$-actin was used as an internal control. The sequences of the primers used were as follows: Fas forward, 5'-AGA AGG GAA GGA GTA CAC TAC GAC-3' and reverse, 5'-TGC ACT TGG TAT TCT GGG TCC-3'; Bax forward, 5'-GTT GCC CTC TTC TAC TTT GC-3' and reverse, 5'-ATG GTC ACT GTC TGC CAT G-3'; $\beta$-actin forward, 5'-ACT GGC ATT GTG ATG GAC TC-3' and reverse, 5'-CAG CAC TGT GTT GGC ATA GA-3'. The samples were analyzed by gel electrophoresis $(1.5 \%)$. The DNA bands were examined using a Gel Documentation System (Model Gel Doc 2000; Bio-Rad, Hercules, CA, USA).

Statistical analysis. All the values are expressed as the mean \pm SE. Statistical analysis of the data was performed using Student's t-test and ANOVA. $\mathrm{P}<0.05$ was considered to indicate a statistically significant difference.

\section{Results}

Effect of electroacupuncture at the Baihui (DU20) and Shenting (DU24) acupoints on neurological deficits and infarct volumes in cerebral I/R injured rats. The neuroprotective effect of electroacupuncture at the Baihui and Shenting acupoints was evaluated by determining the neurological deficit scores. As hypothesized, rats in the SC group did not exhibit any manifestations of neurological deficits (Fig. 1), whereas all the rats in the IC and EA groups had clear symptoms of cerebral injury. However, electroacupuncture significantly improved the neurological deficit scores $(\mathrm{P}<0.05$, EA vs. IC group; Fig. 1). To further verify these results, we evaluated the effect of electroacupuncture on cerebral infarction. As shown in Fig. 2, electroacupuncture treatment 

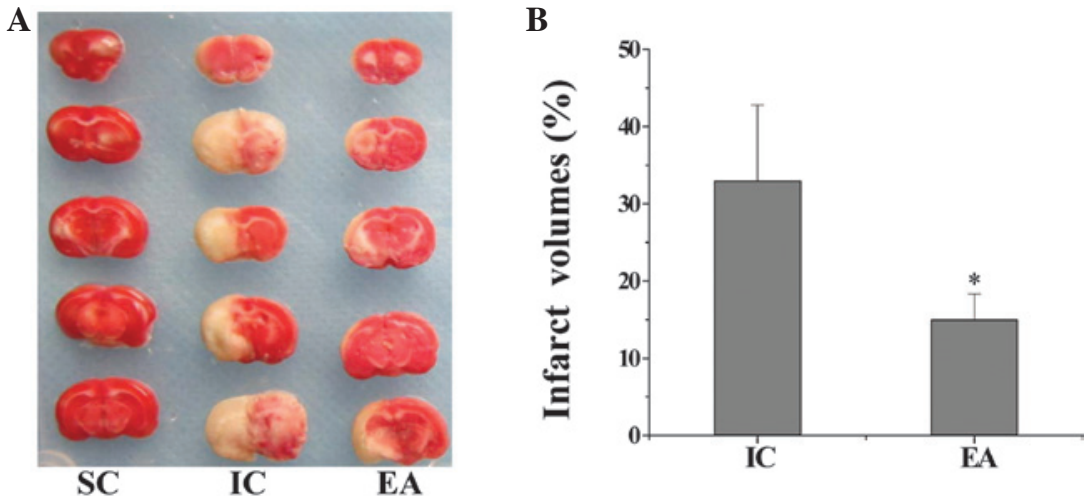

Figure 2. Effect of electroacupuncture on cerebral infarction in cerebral ischemia-reperfusion (I/R) injured rats. (A) Following completion of the experiment, cerebral tissues from the rats of each group were sectioned into 2-mm coronal slices and then stained using 2,3,5-triphenyl tetrazolium chloride (TTC). Images were captured using a high-resolution digital camera. Images are representative of three independent experiments. (B) Infarct volume was quantified with the Motic Med 6.0 as a percentage of the total brain volume. Data are presented as averages with SE (error bars) from 4 individual rats in each group. " $\mathrm{P}<0.05$ vs. the ischemia control (IC) group. SC, sham operation control group; EA, electroacupuncture group.

A

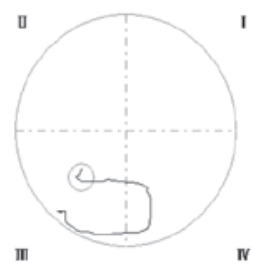

SC

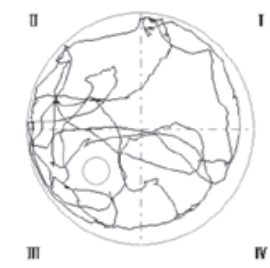

IC

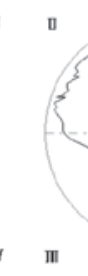

III

.

EA
B

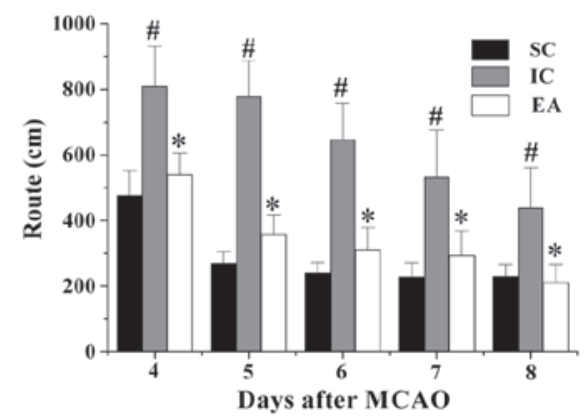

D

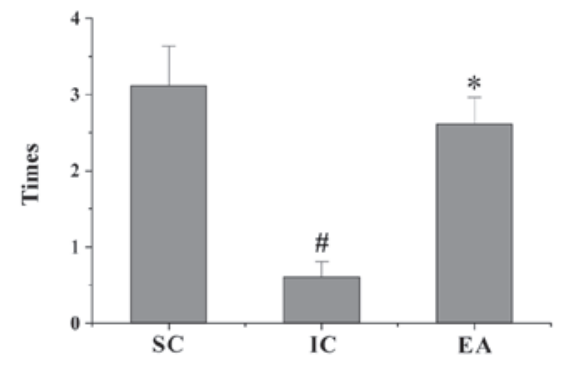

C

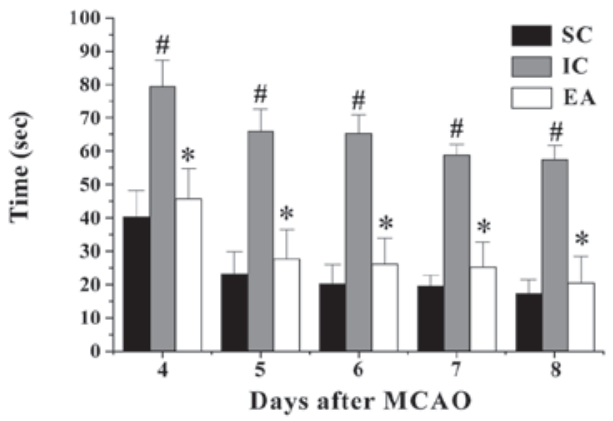

Figure 3. Effects of electroacupuncture on cognitive impairment in cerebral ischemia-reperfusion (I/R) injured rats. The learning and memory ability of rats was determined using a Morris water maze test on days 4-9 following I/R injury. (A) Tracing images from the Morris water maze test. (B) Length of the route and (C) time taken for rats to find the platform (within $90 \mathrm{sec}$ ). (D) The number of times the rats passed through the area in which the platfrom was located were determined on the 9 th day after I/R injury. Data are presented as averages with SE (error bars) from 15 individual rats in each group. ${ }^{\#} \mathrm{P}<0.05$ vs. the sham operation control (SC) group; ${ }^{*} \mathrm{P}<0.05$ vs. the ischemia control (IC) group. EA, electroacupuncture group; MCAO, middle cerebral artery occlusion.

significantly reduced cerebral infarct volumes in cerebral $\mathrm{I} / \mathrm{R}$ injured rats $(\mathrm{P}<0.05$, EA vs. IC group). These results indicate that electroacupuncture at Baihui (DU20) and Shenting (DU24) may have therapeutic efficacy against cerebral I/R injury.

Electroacupuncture ameliorates cognitive impairment in cerebral I/R injured rats. To evaluate the effect of electroacupuncture on cognitive function, a Morris water maze test was performed on days 4-9 following MCAO surgery. As shown in Fig. 3, the latency and route for rats in the IC group to reach the hidden platform in the Morris water maze test markedly increased, whereas the number of times that rats crossed the location of the platform was significantly decreased compared with rats in the $\mathrm{SC}$ group $(\mathrm{P}<0.05)$, indicating that cerebral I/R injury resulted in cognitive impairment. However, electroacupuncture significantly decreased the latency and route length, and increased the number of times the platform was crossed in the Morris water maze test $(\mathrm{P}<0.05$ vs. the IC group; Fig. 3 ). Collectively, these data suggest that electroacupuncture at the Baihui and Shenting acupoints ameliorate cognitive impairment in cerebral I/R injured rats. 
A

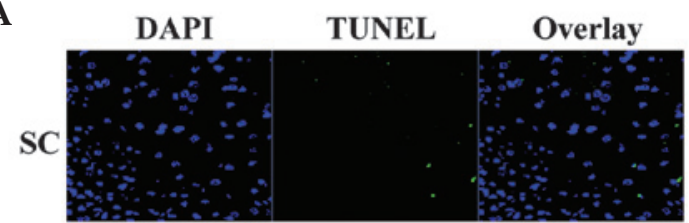

IC

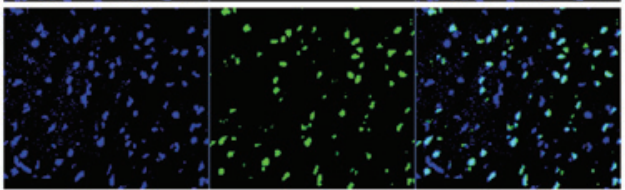

EA

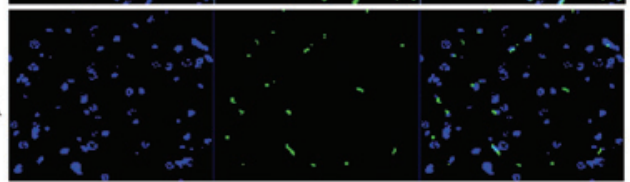

$\mathbf{B}$

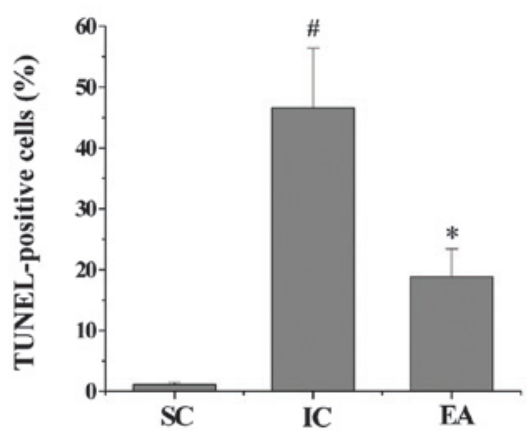

Figure 4. Effect of electroacupuncture on cerebral cell apoptosis in cerebral ischemia-reperfusion (I/R) injured rats. Following completion of the experiment, cerebral tissues from each group $(n=4)$ were processed using a TUNEL assay. The nuclei of all the cells were visualized by DAPI staining and the green fluorescence of apoptotic cells was detected using a confocal fluorescence microscope. Apoptotic cells were counted at four randomly selected microscopic fields (magnification, x200). The apoptotic rate was calculated as the ratio of green-stained cells to the total number of blue DAPI-stained cells. Data are presented as averages with SE (error bars). ${ }^{\#} \mathrm{P}<0.05$ vs. the sham operation control (SC) group; ${ }^{*} \mathrm{P}<0.05$ vs. the ischemia control (IC) group. EA, electroacupuncture group; TUNEL, terminal deoxynucleotidyl-transferase-mediated dUTP nick end labeling.

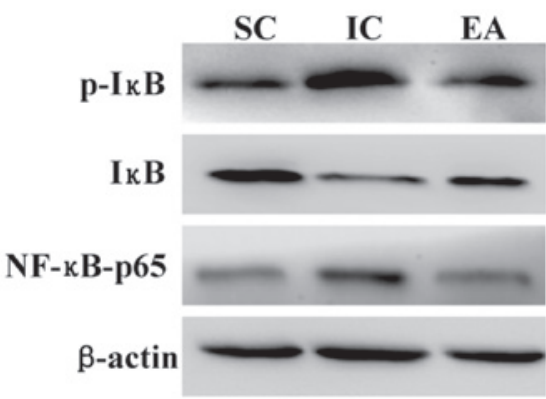

Figure 5. Effect of electroacupuncture on the activation of $\mathrm{NF}-\kappa \mathrm{B}$ pathway in cerebral ischemia-reperfusion $(\mathrm{I} / \mathrm{R})$ injured rats. The protein expression

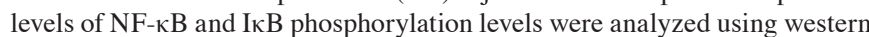
blot analysis. $\beta$-actin was used as an internal control. Data are representative of 4 individual rats in each group. SC, sham operation control group; IC, ischemia control group; EA, electroacupuncture group.

Electroacupuncture inhibits cerebral cell apoptosis in cerebral $I / R$ injured rats. Cognitive impairment is known to be strongly associated with neuronal cell apoptosis (33); therefore, we evaluated the effect of electroacupuncture on cell apoptosis in

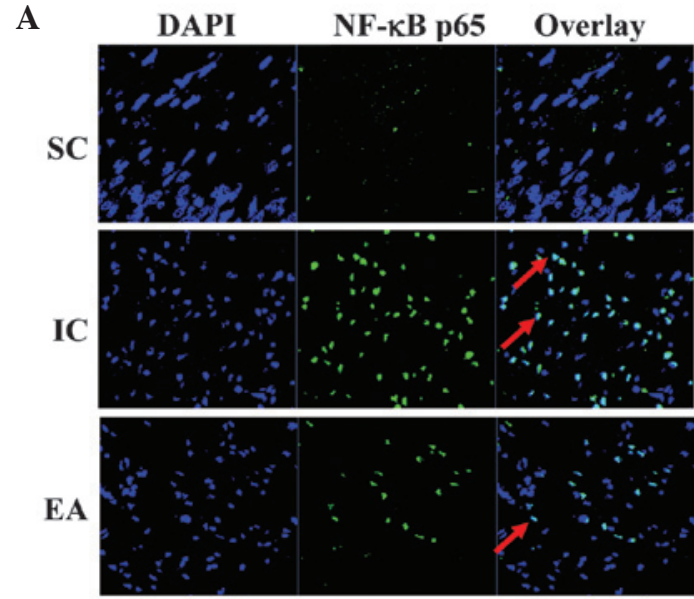

$\mathbf{B}$

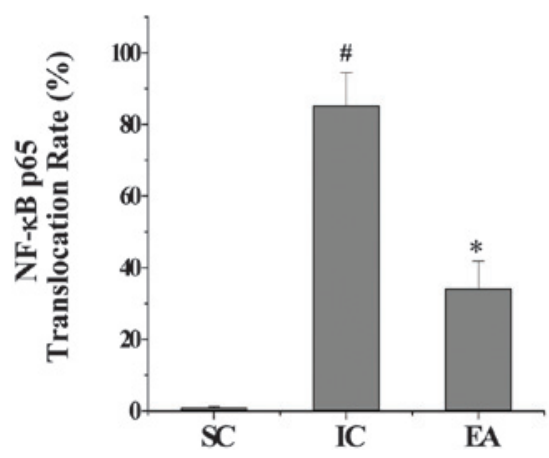

Figure 6. Effect of electroacupuncture on the nuclear translocation of the $\mathrm{NF}-\kappa \mathrm{B}$ p65 subunit in cerebral ischemia-reperfusion (I/R) injured rats. Following completion of the experiment, cerebral tissues were processed for an immunofluorescence assay. The NF- $\kappa \mathrm{B}$ p 65 subunit was visualized by immunofluorescent staining (green) and the cells were counterstained with DAPI (blue). NF- $\mathrm{B}$ nuclear translocation was indicated by the co-localization of the p65 subunit with DAPI (red arrows). Images were captured using a confocal fluorescence microscope (Leiss LSM 710; magnification, x200). Images are representative of 4 individual rats in each group. ${ }^{*} \mathrm{P}<0.05$ vs. the sham operation control (SC) group; ${ }^{*} \mathrm{P}<0.05$ vs. the ischemia control (IC) group. EA, electroacupuncture group.

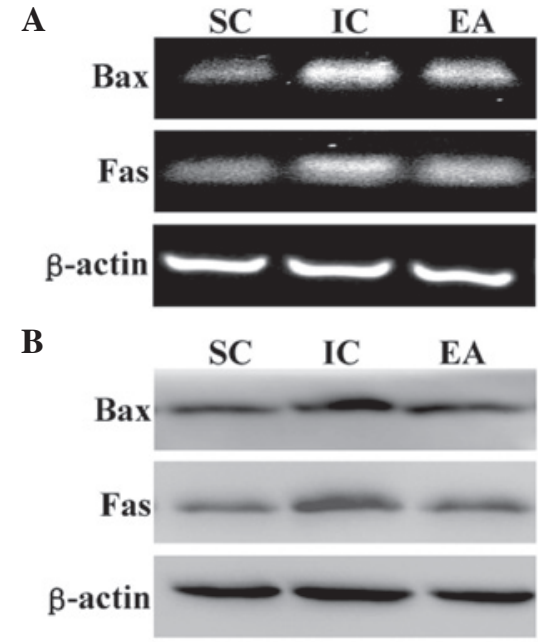

Figure 7. Effect of electroacupuncture on the expression of Bax and Fas in cerebral ischemia-reperfusion (I/R) injured rats. (A) mRNA levels of Bax and Fas in ischemic cerebral tissues were determined using reverse transcription polymerase chain reaction (RT-PCR). (B) Protein expression levels of Bax and Fas were analyzed using western blot analysis. $\beta$-actin was used as an internal control for RT-PCR and western blot analysis. Data are representative of 4 individual rats in each group. SC, sham operation control group; IC, ischemia control group; EA, electroacupuncture group. 
ischemic cerebral tissues using a TUNEL assay. As shown in Fig. 4, the percentage of TUNEL-positive cells in the SC and IC groups was $1.2 \pm 0.3$ and $46.7 \pm 9.7 \%$, respectively $(\mathrm{P}<0.05)$, indicating that $\mathrm{I} / \mathrm{R}$ injury significantly promoted cerebral cell apoptosis. However, the percentage of TUNEL-positive cells in the EA group was $18.9 \pm 4.5 \%(\mathrm{P}<0.05$ compared with the IC group), which demonstrates that electroacupuncture significantly inhibited the I/R-induced apoptosis of neuronal cells. This suggests that electroacupuncture at the Baihui and Shenting acupoints inhibits ischemia-mediated cerebral cell apoptosis.

Electroacupuncture inhibits the $N F-\kappa B$ signaling pathway in cerebral $I / R$ injured rats. Since the activation of $N F-\kappa B$ signaling is important in cerebral cell apoptosis in ischemic stroke, we examined the effect of electroacupuncture on the $\mathrm{NF}-\kappa \mathrm{B}$ pathway in ischemic cerebral tissues. As shown in Fig. 5, the protein expression of NF- $\kappa \mathrm{B}$ p 65 and the I $\kappa \mathrm{B}$ phosphorylation levels were significantly increased in the IC group compared with those in the SC group, suggesting that I/R injury significantly activates NF- $\mathrm{B}$ signaling. However, electroacupuncture neutralized the effect of model construction, suppressing NF- $\mathrm{B}$ protein expression and $\mathrm{I} \kappa \mathrm{B}$ phosphorylation in ischemic cerebral tissues. To verify these observations, immunofluorescence staining was performed to examine the nuclear translocation of $N F-\kappa B$, a critical step for $N F-\kappa B$ activation. As shown in Fig. 6, cerebral I/R injury significantly induced the nuclear translocation of the NF- $\mathrm{NB}$ p65 subunit; however, this was inhibited by electroacupuncture. Taken together, these findings indicate that the anti-apoptotic activity of electroacupuncture in cerebral I/R injured rats was mediated by inhibition of the $\mathrm{NF}-\kappa \mathrm{B}$ pathway.

Electroacupuncture downregulates the apoptotic Fas/Bax genes in cerebral I/R injured rats. Apoptosis is highly regulated by various factors, including Bax and Fas. Additionally, pro-apoptotic Bax and Fas are important downstream target genes of the NF- $\kappa \mathrm{B}$ signaling pathway. To further investigate the mechanism of the anti-apoptotic activity of electroacupuncture, we investigated the mRNA levels and protein expression of Fas and Bax in ischemic cerebral tissues using RT-PCR and western blot analysis, respectively. As shown in Fig. 7, cerebral I/R injury markedly enhanced Bax and Fas expression at transcriptional and translational levels; however, this was neutralized by electroacupuncture.

\section{Discussion}

Survivors of stroke frequently present with cognitive impairment, which severely affects their quality of life. Cognitive impairment is strongly associated with neuronal cell apoptosis, which is tightly regulated by various intracellular signal transduction cascades, including the $\mathrm{NF}-\kappa \mathrm{B}$ pathway $(34,35)$. Previous studies have demonstrated that $\mathrm{NF}-\kappa \mathrm{B}$ signaling is activated in post-stroke cognitive impairment, suggesting that the NF- $\kappa \mathrm{B}$ pathway may be a major target for the treatment of impaired cognition (24). In TCM, acupuncture has been used as a complementary and alternative method for thousands of years. Numerous studies have demonstrated the clinical efficacy of acupuncture in stroke and cognitive impairment (30). According to TCM, Baihui (DU20) and Shenting (DU24) are located on the Du Meridian, which is considered to be important in the nervous system. Consequently, these two acupoints are commonly used in China to clinically treat cognitive impairment (30). However, the precise mechanism of its therapeutic effect on impaired cognition remains unclear.

In the present study, a focal cerebral I/R rat model was constructed and electroacupuncture at the Baihui and Shenting acupoints was shown to have a neuroprotective effect, as it significantly ameliorated neurological deficits and reduced cerebral infarct volume. Additionally, a Morris water maze test revealed that electroacupuncture improved learning and memory ability in cerebral I/R injured rats, demonstrating its therapeutic efficacy against post-stroke cognitive impairment. Furthermore, the NF- $\kappa \mathrm{B}$ pathway was identified to be activated after cerebral I/R injury, which was consistent with the results of previous studies (36). However, electroacupuncture significantly suppressed $\mathrm{NF}-\kappa \mathrm{B}$ signaling in ischemic cerebral tissues. The inhibitory effect of electroacupuncture on $\mathrm{NF}-\kappa \mathrm{B}$ activation led to the inhibition of cerebral cell apoptosis. Apoptosis is activated through two major pathways; in the intrinsic pathway, death signals are integrated at the level of the mitochondria, while in the extrinsic pathway, death signals are mediated through cell surface receptors. Both pathways eventually lead to the activation of caspases and nucleases, resulting in the destruction of the cell. Bax and Fas, two critical downstream target genes of the $\mathrm{NF}-\kappa \mathrm{B}$ pathway, exert their pro-apoptotic function via the intrinsic and extrinsic pathways, respectively (37). As hypothesized, electroacupuncture significantly downregulated the expression of Bax and Fas at the transcriptional and translational levels.

In conclusion, the present study showed for the first time that electroacupuncture at the Baihui (DU20) and Shenting (DU24) acupoints has a therapeutic function in ischemic stroke and impaired cognition via inhibition of NF- $\mathrm{NB}-$ mediated neuronal cell apoptosis. These results suggest that electroacupuncture may be a potential therapeutic modality for the treatment of post-stroke cognitive impairment.

\section{Acknowledgements}

This study was sponsored by the International S\&T Cooperation Program of China (ISTCP Program; No. 2011DFG33240), the key International S\&T Cooperation Program of Fujian Science and Technology Department (No. 2010I0007) and 'Twelfth five-year' national Technology Support Project (No. 2013BAI10B01).

\section{References}

1. Jokinen H, Kalska H, Mäntylä R, et al: Cognitive profile of subcortical ischaemic vascular disease. J Neurol Neurosurg Psychiatry 77: 28-33, 2006.

2. Lindeboom $J$ and Weinstein H: Neuropsychology of cognitive ageing, minimal cognitive impairment, Alzheimer's disease, and vascular cognitive impairment. Eur J Pharmacol 490: 83-86, 2004.

3. Nyenhuis DL, Gorelick PB, Geenen EJ, et al: The pattern of neuropsychological deficits in Vascular Cognitive Impairment-No Dementia (Vascular CIND). Clin Neuropsychol 18: 41-49, 2004.

4. Sachdev PS, Brodaty H, Valenzuela MJ, et al: The neuropsychological profile of vascular cognitive impairment in stroke and TIA patients. Neurology 62: 912-919, 2004. 
5. Mok V, Chang C, Wong A, et al: Neuroimaging determinants of cognitive performances in stroke associated with small vessel disease. J Neuroimaging 15: 129-137, 2005.

6. Mok VC, Wong A, Lam WW, et al: Cognitive impairment and functional outcome after stroke associated with small vessel disease. J Neurol Neurosurg Psychiatry 75: 560-566, 2004.

7. Haring HP: Cognitive impairment after stroke. Curr Opin Neurol 15: 79-84, 2002.

8. Alvarez-Sabín J and Román GC: Citicoline in vascular cognitive impairment and vascular dementia after stroke. Stroke 42 (Suppl 1): S40-S43, 2011.

9. Hachinski V and Munoz D: Vascular factors in cognitive impairment - where are we now? Ann NY Acad Sci 903: 1-5, 2000 .

10. Tatemichi TK, Desmond DW, Stern Y, et al: Cognitive impairment after stroke: frequency, patterns, and relationship to functional abilities. J Neurol Neurosurg Psychiatry 57: 202-207, 1994.

11. Desmond DW, Moroney JT, Paik MC, et al: Frequency and clinical determinants of dementia after ischemic stroke. Neurology 54: 1124-1131, 2000.

12. Mattson MP: Apoptosis in neurodegenerative disorders. Nat Rev Mol Cell Biol 1: 120-129, 2000.

13. Nakka VP, Gusain A, Mehta SL and Raghubir R: Molecular mechanisms of apoptosis in cerebral ischemia: multiple neuroprotective opportunities. Mol Neurobiol 37: 7-38, 2008.

14. Broughton BR, Reutens DC and Sobey CG: Apoptotic mechanisms after cerebral ischemia. Stroke 40: e331-e339, 2009.

15. Cory S and Adams JM: The Bcl2 family: regulators of the cellular life-of-death switch. Nat Rev Cancer 2: 647-656, 2002.

16. Borner $\mathrm{C}$ : Bcl-2 family members: integrators of survival and death. Biochim Biophys Acta 1644: 71-72, 2004.

17. Baeuerle PA and Baltimore D: NF-kappa B: ten years after Cell 87: 13-20, 1996.

18. Taglialatela G, Robinson R and Perez-Polo JR: Inhibition of nuclear factor kappa B (NFkappaB) activity induces nerve growth factor-resistant apoptosis in PC12 cells. J Neurosei Res 47: 155-162, 1997.

19. Middleton G, Hamanoue M, Enokido Y, et al: Cytokine-induced nuclear factor kappa B activation promotes the survival of developing neurons. J Cell Biol 148: 325-332, 2000.

20. Goodman Y and Mattson MP: Ceramide protects hippocampal neurons against excitotoxic and oxidative insults, and amyloid beta-peptide toxicity. J Neurochem 66: 869-872, 1996

21. Mattson MP, Goodman Y, Luo H, et al: Activation of NF-kappaB protects hippocampal neurons against oxidative stress-induced apoptosis: evidence for induction of manganese superoxide dismutase and suppression of peroxynitrite production and protein tyrosine nitration. J Neurosci Res 49: 681-697, 1997.

22. Grilli M, Pizzi M, Memo M and Spano P: Neuroprotection by aspirin and sodium salicylate through blockade of NF-kappaB activation. Science 274: 1383-1385, 1996.
23. Won SJ, Ko HW, Kim EY, et al: Nuclear factor kappa B-mediated kainite neurotoxicity in the rat and hamster hippocampus. Neuroscience 94: 83-91, 1999.

24. van der Kooij MA, Nijboer CH, Ohl F, et al: NF-kappaB inhibition after neonatal cerebral hypoxia-ischemia improves long-term motor and cognitive outcome in rats. Neurobiol Dis 38: 266-272, 2010.

25. Wu JN: A short history of acupuncture. J Altern Complement Med 2: 19-21, 1996.

26. Hu HH, Chung C, Liu TJ, et al: A randomized controlled trial on the treatment for acute partial ischemic stroke with acupuncture. Neuroepidemiology 12: 106-113, 1993.

27. Jansen G, Lundeberg T, Kjartansson J and Samuelson UE: Acupuncture and sensory neuropeptides increase cutaneous blood flow in rats. Neurosci Lett 97: 305-309, 1989.

28. Johansson K, Lindgren I, Widner H, et al: Can sensory stimulation improve the functional outcome in stroke patients? Neurology 43: 2189-2192, 1993.

29. Magnusson M, Johansson K and Johansson BB: Sensory stimulation promotes normalization of postural control after stroke. Stroke 25: 1176-1180, 1994.

30. Zhao L, Zhang H, Zheng Z, et al: Electroacupuncture on the head points for improving gnosia in patients with vascular dementia. J Tradit Chin Med 29: 29-34, 2009.

31. Chen LP, Wang FW, Zuo F, et al: Clinical research on comprehensive treatment of senile vascular dementia. J Tradit Chin Med 31: 178-181, 2011.

32. Chen AZ, Lin ZC, Lan L, et al: Electroacupuncture at the Quchi and Zusanli acu points exerts neuroprotective role in cerebral ischemia- reperfusion injured rats via activation of the PI3K/Akt pathway. Int J Mol Med 30: 791-796, 2012.

33. Zhang GZ, Liu AL and Zhou YB: Panax ginseng ginsenoside$\mathrm{Rg} 2$ protects memory impairment via-anti-apoptosis in a rat model with vascular dementia. J Ethnopharmacol 115: 440-448, 2008.

34. Sarnico I, Lanzillotta A and Benarese M: NF-kappaB dimers in the regulation of neuronal survival. Int Rev Neurobiol 85: 351-362, 2009.

35. Freudenthal R, Romano A and Routtenberg A: Transcription factor NF-kappaB activation after in vivo perforant path LTP in the mouse hippocampus. Hippocampus 14: 677-683, 2004.

36. Zhang W, Potrovita I, Tarabin V, et al: Neuronal activation of $\mathrm{NF}-\mathrm{kB}$ contributes to cell death in cerebral ischemia. J Cerebral Blood F Met 25: 30-34, 2005.

37. Kumar A, Takada Y, Boriek AM and Aggarwal BB: Nuclear factor-kB: its role in health and disease. J Mol Med (Berl) 82: 434-448, 2004. 